\title{
Electric grid reliability research
}

Vivian Sultan ${ }^{*}$ (D) and Brian Hilton

\author{
* Correspondence: vivian.sultan@ \\ icloud.com \\ Claremont Graduate University, \\ Claremont, CA 91711, USA
}

\begin{abstract}
Background: Energy informatics (EI) is the area of research that addresses the application of technology to resolve complex problems in the energy domain. Goebel et al. (BISE 6:25-31, 2014) provided an El research framework to encompass all aspects of El research. Due to rapid El improvements, many current research areas have not been incorporated into this framework. Specifically, this research posits that grid reliability is an underrepresented research area and should be incorporated into the framework. The rapidly changing nature of energy generation, and new developments in the electric-power network, are driving the need for grid reliability research. The goal of this paper is to extend the El research framework offered by the Goebel et al. through a systematic review of current literature.

Methods: This literature review includes current publications (2015-2017) in power utility and technical reference libraries together with the earlier foundational El papers.

Results: The systematic literature review is based on a broad automated search of appropriate digital sources (IEEE Xplore and the Web of Science) using relevant search terms. The paper also details the components of grid reliability research as well as their accompanying use cases.

Conclusion: The expanded El research framework presented in this literature review should help researchers identify areas for future research endeavors. In the extended El research framework, service reliability is now included as a third research component adding to the existing energy efficiency and renewable-energy supply components.
\end{abstract}

Keywords: Energy informatics, Grid reliability, El research framework

\section{Background}

Energy informatics (EI) research concerns the use of information and communication technologies to address energy challenges (Watson and Boudreau 2011) and to inform the application of technology to resolve complex problems in the energy domain. The U.S. Department of Energy, Office of Energy Efficiency and Renewable Energy (2017) classified challenges within the electricity-grid domain into two types: (1) transmission-system challenges and (2) distribution-system challenges. Transmissionsystem challenges include grid operations, grid reliability, grid stability, models and codes, grid operators, and utilities. Distribution-system challenges includes voltage and volt-ampere reactivity regulation, unintentional islanding, power quality, protection coordination, distribution modeling, visibility and control, codes, and standards.

Goebel et al. (2014) stated that energy efficiency and renewable-energy supply are the two principal types of research movements within the energy domain. Energy efficiency research involves studying individual incentives and behavioral dynamics to

(c) The Author(s). 2019 Open Access This article is distributed under the terms of the Creative Commons Attribution 4.0 International License (http://creativecommons.org/licenses/by/4.0/), which permits unrestricted use, distribution, and reproduction in any medium, provided you give appropriate credit to the original author(s) and the source, provide a link to the Creative Commons license, and indicate if changes were made. 
influence electricity consumers' usage behavior. This first type drives the evolution of smart energy-saving systems. The second type of research, renewable-energy supply, seeks to resolve challenges arising in the integration of such renewable sources of energy as wind and solar power into the electric grid. This, in turn drives, the advancement of smart grids.

This research paper discusses an understudied research area within EI, grid reliability. Given the rapidly changing nature of energy generation, new developments of the electric power network, the incorporation of distributed energy resources into the grid, and circuit and equipment overloads, grid reliability research has been underwhelming. According to Goebel et al. (2014), energy efficiency and the renewable-energy supply are the two principal types of research movements in the energy domain. In their EI research framework, Goebel et al. considered grid reliability a subtopic, one of the many segments underlying the renewable-energy-supply research theme. Specifically, reliability was a segment under renewable-energy research. However, rapidly shifting challenges within the electric utility industry suggest that grid-reliability research should be classified as a new and separate area of research.

Grid reliability has several definitions. The North American Electric Reliability Corporation defined reliability as "the degree to which the performances of the elements of the electric system result in power being delivered to consumers within accepted standards and in the amount desired" (Hirst and Kirby 2000, p 7). Osborn and Kawann (2001) viewed reliability as "the ability of the power system components to deliver electricity to all points of consumption, in the quantity and with the quality demanded by the customer" ( $\mathrm{p} 2$ ). Reliability is measured by outage indices as illustrated by the Institute of Electrical and Electronics Engineers' Standard 1366. To facilitate a unified view of grid reliability, a definition is proposed: "the ability of the electric grid to deliver electricity to customers without degradation or failure." The argument is that today's power systems cannot accommodate, for instance, significant variable distributed energy generation without failure (U.S. Department of Energy 2015).

Grid reliability aims to address challenges and remove barriers to integrating high penetration of distributed-energy generation at the transmission and distribution levels (U.S. Department of Energy, Office of Energy Efficiency and Renewable Energy 2017). The subject includes many unaddressed questions: What makes the grid reliable? Why might reliability degrade? How do ongoing changes in grid use impose such a risk? What can we do to improve grid reliability? The U.S. Department of Energy has recently released its electric-grid reliability study, recommending prioritization of developments for grid resiliency and reliability (Profeta 2017). Additionally, in the same month, the U.S. Department of Energy, Office of Electricity announced an initial investment of nearly $\$ 900,000$ to address power systems' risks and uncertainty, enabling academic research in the United States (Laezman 2017).

The goal of this paper is to restructure the Goebel et al. (2014) EI research framework through a systematic literature review to produce an updated framework highlighting grid-reliability research, all of its components, and its additional use cases.

The rest of the paper is organized as follows. The second section presents research importance. The third section describes the methodology used to conduct the systematic literature review. The fourth section outlines the results of the review, proposes an 
enhanced EI framework, and presents a bibliography of foundational papers. Finally, the fifth section offers conclusions.

\section{Research importance}

Due to a rise in electricity use resulting from such new technologies as electric vehicles and circuit and equipment overloads, a significant number of publications from research organizations, governmental bodies, and utility companies have focused on understanding grid reliability, fault causes, and power outages. The National Academies of Sciences, Engineering, and Medicine (2017) recently published "Enhancing the Resilience of the Nation's Electricity System" in response to Congress's call for an independent assessment to "conduct a national-level comprehensive study on the future resilience and reliability of the nation's electric power transmission and distribution system." In addition, the National Academies of Sciences, Engineering, and Medicine established a committee to conduct the relevant research. Throughout this report, the committee highlighted all elements of grid reliability, resilience, and the risks of the system-wide failure that will grow as the structure of the power industry becomes more atomized and complex and laid out a wide range of actions to improve the U.S. power system's resilience. Analytics (including machine learning, data mining, and other artificial-intelligence techniques) will play a very important role in response to the predicted attacks on the electric grid, failures, or other impairments due to their ability to generate real-time recommendations (National Academies of Sciences, Engineering, and Medicine 2017).

In another exemplar research, Shawn Adderly (2016) examined U.S. Department of Energy's power outage data from 2002 to 2013 and investigated reliability trends. The research objective was to assess the correlation between utilities' reliability and grid-investment projects such as the deployment of smart-grid assets. Using the deployment of smart meters as a proxy for grid investments, Adderly concluded that the increase of smart meters correlated strongly with a decrease in the frequency of outages. The author acknowledged that due to the presence of confounding variables, the reduction in power outage could not be attributed to any specific smart-grid investment project.

Several studies attempt to understand grid reliability. Steve Mitnick's (2015) report prepared for the Electric Markets Research Foundation, another important resource, explains the reasons for concerns about grid reliability. The author suggests that distributed energy resources must be carefully incorporated into the grid to minimize grid-reliability risk. Another relevant study conducted by the Lawrence Berkeley National Lab (Eto et al. 2012) highlighted the fact that reliability-data trends might not improve due to the addition of smart-grid technology because automated outage-management systems may report service interruptions more accurately. Since the study was based on a sample of reliability data from several utilities, the authors did not attempt to make claims about overall U.S. power reliability.

With respect to the power-outage-causes study domain, the majority of the outages in the United States are the result of events that occur on the grid's distribution side. Few outages are caused by the external factors. The three main causes for electrical outages are (1) hardware and technical failures, (2) environmental incidents, and (3) 
human errors. Among hardware and technical failures, outages are experienced due to equipment overload and short circuits, to name a few (Westar Energy 2017; Diesel Service and Supply 2017; Rocky Mountain Power 2017). These failures are often attributed to unmet peak demand, outdated equipment, and malfunctions of backup power systems (Chayanam 2005).

Environmental incidents, the largest portion of power outage causes, can be classified into two distinct categories: weather and other factors. Such publications as Wisconsin Public Service (2017) have highlighted the weather-related causes of power outages. The Edison Electric Institute states that $70 \%$ of U.S. power outages are weather related (as cited in Campbell 2012). Kenward and Raja (2014) analyzed power outage data over a 28-year period and noted that between 2003 and 2012, 80\% of all outages were caused by weather. Similarly, Campbell (2012) highlighted the damage to the electrical grid caused by seasonal storms, rain, and high winds.

Besides weather, other external forces create power outages. As a byproduct of heavy weather patterns, falling tree branches disrupt the flow of electricity (National Academies of Sciences, Engineering, and Medicine 2017). Animals are another culprit of power disruption. The Edison Electric Institute study also indicated that animals, such as large birds, contacting power lines accounted for $11 \%$ of U.S. outages (as cited in Campbell 2012).

Human-error incidents are the last cause of power outages. Chayanam (2005) indicated that training is essential for technicians and staff to battle outages with proper maintenance procedures. This ensures a reduction in the frequency of power outages. Car accidents are another major source of power outages (Wisconsin Public Service 2017).

In a 2013 white paper entitled "the Smart Grid Investment Grant Program Progress Report," the Department of Energy highlighted reliability improvements observed through decreasing reliability indices and highlighted that such projects as automated feeder switching were able to reduce the frequency of outages. No statistics were shown in the report to demonstrate the correlation between reliability indices and spending. However, the study identified the progress made by utilities as a result of receiving federal funding (U.S. Department of Energy 2013).

Interrupted power supply is no longer a mere inconvenience. As the duration and spatial extent of electrical outages increases, costs and inconvenience grow. Critical social services such as medical care, police and other emergency services, and communications systems rely upon electricity to function at minimally acceptable levels. Failures can bring catastrophic outcomes; lives can be lost. We must better understand the causes to be more ready to implement redundancy and resilience in the electric grid.

To heed this call, this paper presents a systematic grid-reliability literature review to help understand the topic's current knowledge base. A systematic literature review is a particularly influential tool in the hands of researchers because it allows a scholar to gather and recap all the information about research in a specific field (Spanos and Angelis 2016). In this first systematic grid-reliability literature review, the focus is on the different grid-reliability topics and their specific characteristics. This article should enrich future literature reviews while integrating the most current articles into the body of knowledge. 


\section{Methodology}

The systematic literature review offered in this paper follows the three stages in a systematic review: the Planning Stage, the Conducting Stage, and the Reporting Stage (Kitchenham 2004; Kitchenham and Charters 2007).

The first step is the identification of a need for a systematic review. As described in the previous two sections, although several studies have investigated electric-grid reliability, these studies should be summarized to update the current knowledgebase. Therefore, the urgent need for a systematic literature review providing solid foundations and equipping researchers with pertinent information is clear.

The second step is the development of the review protocol. This section presents the research questions, search strategy, inclusion/exclusion criteria, quality-assessment criteria, and the data extracted from the studies.

Defining research questions is an essential step in every systematic review. By answering the following questions, the literature review can accomplish its aim.

1. How many research studies have examined electric-grid reliability?

2. What are the types of reliability-research questions?

3. What are the studies' results?

4. What research methods are used?

To conduct the systematic literature review, it was decided to do a broad automated search, a method that includes the selection of the most appropriate digital sources (digital libraries and indexing systems) and the determination of the search terms (Spanos and Angelis 2016). The digital libraries of IEEE Xplore and the Web of Science were selected for the systematic review. The Web of Science database provides a wide breadth and depth, whereas the IEEE database provides more narrowly focused and very recent research. These searches relied on papers' titles to avoid receiving duplicate or irrelevant papers as search results. The following search strings were used.

\section{IEEE Xplore Boolean/Phrase}

((((“"Document Title”:Electricity Reliability) OR “Document Title”:Electric Grid Reliability) OR "Document Title":Power System Reliability) OR “Document Title":Electric Circuit Reliability) OR “Document Title”:Power Outage Research)

refined by.

Content Type: Conference Publications Journals \& Magazines Books \& eBooks Year: 2015-2017

The year range is limited to those 3 years to ensure the search captures the reliability impact of integrating distribution-energy resources into the electric grid.

\section{Web of Science Boolean/Phrase}

TITLE:(Electricity Reliability) OR TITLE:(Electric Grid Reliability) OR TITLE:(Power System Reliability) OR TITLE:(Electric Circuit Reliability) OR TITLE:(Power Outage Research) AND TITLE: ("estimate"” OR "assess"”)

refined by.

DOCUMENT TYPES: (PROCEEDINGS PAPER OR ARTICLE) 
Timespan: 2015-2017. Indexes: SCI-EXPANDED, SSCI, A\&HCI, CPCI-S, CPCI-SSH, ESCI.

The inclusion/exclusion selection criteria of any systematic literature review must be distinct and clearly stated (Spanos and Angelis 2016). The following selection criteria were used for the systematic review.

1. Full-article publication (not just an abstract),

2. English-language publication,

3. Year of publication sufficiently recent (since year 2015) to ensure that the reliability impact of integrating distribution energy resources into the electric grid has been captured, and

4. Study relevance.

The following exclusion criterion was used.

1. Duplicate publications (to avoid double counting studies)

The quality-assessment criteria are provided to ensure that all the included studies in a systematic literature review contain an adequate level of quality. The following were the criteria.

- Availability and the description of the data,

- Description of the used methodology, and

- Presentation of the results.

The last task is related to the development of the review protocol and the selection of the data features that will be extracted from the papers found by the search. The data features for this review are as follows.

- Author(s),

- Year of publication,

- Type of paper,

- The research question(s),

- Method(s) used, and

- Result(s).

\section{Results}

This section presents the initial results of the systematic literature review: 209 papers from the IEEE Xplore database and 294 papers from the Web of Science core collection. Table 1 summarizes the characteristics of the documents identified and the counts of papers by year.

The top publishing authors were M Fotuhi-Firuzabad (10 publications), C Singh (8), LF Wang (8), YM Xing (8), and M Benidris (6).

After the excluding duplicate, irrelevant, and low-quality papers, 231 papers remained from the search in the two databases. Table 2 summarizes the search results (503 total paper count from the initial search), the number of non-relevant, the duplicate papers, 
Table 1 Study characteristics

\begin{tabular}{lll}
\hline & IEEE Xplore & Web of science \\
\hline Paper type & 152 & 164 \\
Proceedings and conferences & 146 & \\
IEEE conference publications & 5 & \\
IET conference publications & 1 & 132 \\
VDE conference publications & 57 & \\
Journals and magazines & 1 & \\
CPSS journals and magazines & 40 & 296 \\
IEEE journals and magazines & 16 & 111 \\
IET journals and magazines & 209 & 122 \\
Total & & 61 \\
Year & 66 & 294 \\
2015 & 95 & \\
2016 & 48 & 209 \\
2017 & & \\
Total & & \\
\hline
\end{tabular}

those not-included after the quality assessment, and the final number of relevant papers included in the systematic literature review for analysis.

Preliminary analysis of the relevant papers identified four key types of research question:

1. How does one assess or evaluate reliability of the power grid?

2. How does one improve or enhance the reliability of the electric-power system?

3. How should one plan reliability of the smart grid?

4. What are the impacts of changes, including adding distributed-energy resources, new regulations, and investment projects, on the reliability of the electric-power system?

Research methods within this research domain can be classified either as analytical or as Monte Carlo simulation. Analytical techniques represent the system by a mathematical model and evaluate the reliability indices from this model using mathematical solutions. Monte Carlo simulation methods, on the other hand, estimate the reliability indices by simulating the actual process and the system's random behavior. Simulation treats problems as a series of real experiments. There are advantages and disadvantages to both methods.

Table 2 Database search results

\begin{tabular}{llll}
\hline Database criteria & IEEE Xplore & Web of science & Total count \\
\hline Number of papers found as a result of the search & 209 & 294 & 503 \\
Irrelevant papers & 42 & 41 & 83 \\
Duplicate papers & 7 & 173 & 180 \\
Papers not included due to inadequate quality & 4 & 2 & 6 \\
Not an English-language publication & 2 & 1 & 3 \\
Final number of relevant papers & 154 & 77 & 231 \\
\hline
\end{tabular}


The foremost research methods are modeling and simulation. Simulation modeling is the process of creating and analyzing a prototype of a physical model to predict its performance in the real world. Simulation modeling is used to help researchers understand whether, under what conditions, and in which ways a part could fail and what loads it can withstand. Researchers have used various modeling and simulation tools to perform the analysis, but Monte Carlo simulation is the most dominant research method.

Based on the systematic literature review, the Goebel et al. (2014) EI research framework was extended. The following section illustrates the impact of this extension and highlights the addition of grid reliability research, all of its components, and its use cases.

\section{Energy informatics enhanced research framework}

In the proposed Energy Informatics enhanced research framework (Fig. 1), energy efficiency, renewable-energy supply, and service reliability are the three types of research streams in the energy domain. These streams reflect the topics identified in the systematic literature review. The restructured Goebel et al. (2014) framework includes service reliability as a third research stream in addition to energy efficiency and the renewable-energy supply to distinguish this understudied research area. The first theme, energy efficiency, drives the evolution of smart energy-saving systems. The second theme, renewable-energy supply, drives the advancement of smart grids. Finally, the third additional theme, service reliability, drives smart-grid reliability and resiliency.

In the context of the service-reliability research theme, use cases (a collection of possible scenarios) were classified into four transmission scales: subcontinental, regional,

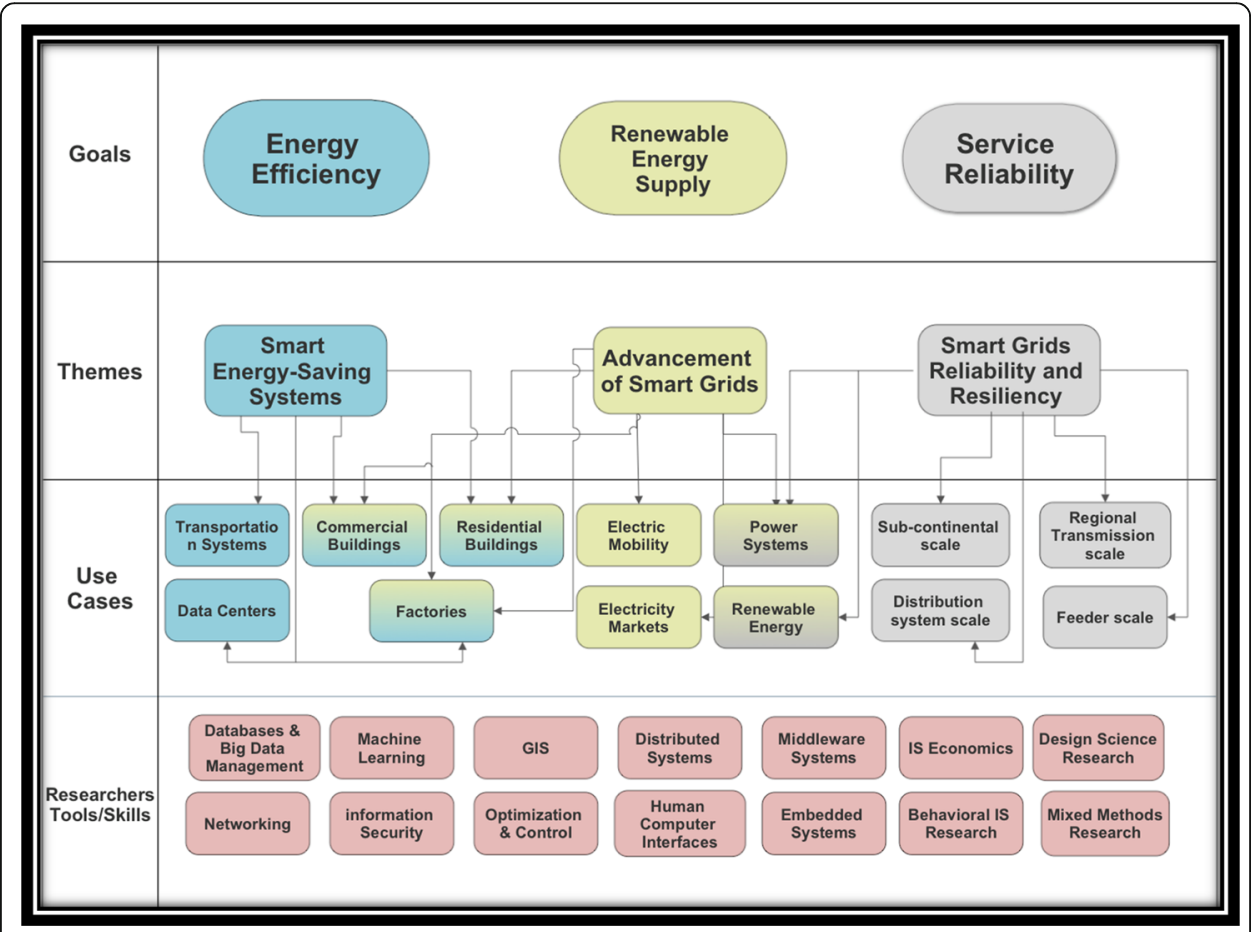

Fig. 1 Energy informatics enhanced research framework-Enriched with reliability research 
local distribution system, and feeder. Power systems and renewable energy can also be viewed as additional use cases due to their impact on grid reliability. The rapidly changing nature of energy generation and the new developments of the electric power network have fueled the rise of grid-reliability research to justify considering it a separate research stream.

The first category of use cases, the subcontinental scale, examines large, relatively self-contained landmasses forming a subdivision of a continent. Within this category, multiple grids, transmission, and distribution systems may exist and be interconnected.

In the second category of use cases, regional transmission, studies examine high-voltage transmission networks that enables power to travel long distances from generating units to substations closer to local end-use customers where the voltage is stepped back down and sent into the distribution system for delivery to consumers. Given the interconnected configuration of the high-voltage grid, events in one place can propagate across the transmission system in seconds or a few minutes, potentially causing cascading blackouts that can affect customers hundreds of miles from the initial disturbance. Outage events on the transmission system can result in large-area impacts (National Academies of Sciences, Engineering, and Medicine (2017).

In the third type of use cases, the electric distribution system moves power from the energy system to the meters of electricity customers. Typically, power is delivered to distribution substations from two or more transmission lines, where its voltage is reduced, and sent to customers over distribution feeders. Although distribution-system outages are more common than those occurring on transmission facilities, their impacts are smaller in scale and generally easier to repair (National Academies of Sciences, Engineering, and Medicine 2017).

The fourth category of use cases includes the feeder scale. Customers on radial systems are exposed to interruption when their feeder experiences an outage. In metropolitan areas, these feeders typically have switches that can be reconfigured to support restoration from an outage or regular maintenance. When a component fails in these systems, customers on unaffected sections of the feeder are switched manually or automatically to an adjacent, functioning, circuit. However, this still exposes critical services such as hospitals or police stations to potential outages, so these facilities are often connected to a second feeder for redundancy (National Academies of Sciences, Engineering, and Medicine 2017).

The definition of service reliability-"the ability of the electric grid to deliver electricity to customers without degradation or failure"-is used to outline the service-reliability research theme. Recent developments such as the integration of distributed-energy resources into the smart grid make information collection, integration, management, and analysis of vital importance. That is why EI has flourished in the research community.

Here, the aim is to contribute to a holistic understanding of problem identification and resolution through the use of tools such as geographic information systems, databases, big-data management, machine learning, information security, and optimization and control. Analytics using these tools could transform the way we think, act, and use energy and help elucidate a problem's root cause, define a solution through data, and implement the solution with continuous monitoring and management. 


\section{Power-system reliability research framework}

In addition to the EI enhanced research framework, Fig. 2 illustrates a framework for power-system reliability founded on the previous literature. The focus within the power-system-reliability research theme can be organized into one of two main types: the bulk-power system (BPS) and the local-distribution system. The first research focus, can be defined as a large interconnected electrical system made up of generation and transmission facilities and their control systems. A BPS does not include facilities used in the local distribution of electric energy. If a BPS is disrupted, the effects are felt in more than one location. In the United States, BPS are managed by the North American Electric Reliability Corporation (National Academies of Sciences, Engineering, and Medicine 2017). Reliability of power supply and system operation, regular evaluations of expected or emerging changes, and system maintenance throughout changes in the electric industry are all possible goals within the BPS research focus.

The local-distribution system, the second research focus, provides power to individual consumer premises. Distribution networks usually consist of distribution substations, primary distribution feeders, distribution transformers, distributors, and service mains (National Academies of Sciences, Engineering, and Medicine 2017). Maintenance and repair of the distribution network, public safety, and operating cost are the possible goals within the local-distribution system research focus.

The combined transmission and distribution network is known as the "power grid" or simply "the grid." North America's BPS involves four different power grids (interconnections). The Eastern Interconnection serves the eastern two thirds of the continental United States and Canada from the Great Plains to the Eastern Seaboard. The Western Interconnection covers the western third of the continental United States, the Canadian provinces of Alberta and British Columbia, and a portion of Baja California Norte in Mexico. The Texas Interconnection includes most of the State of Texas. Finally, the Canadian province of Quebec is served by the fourth North American interconnection.

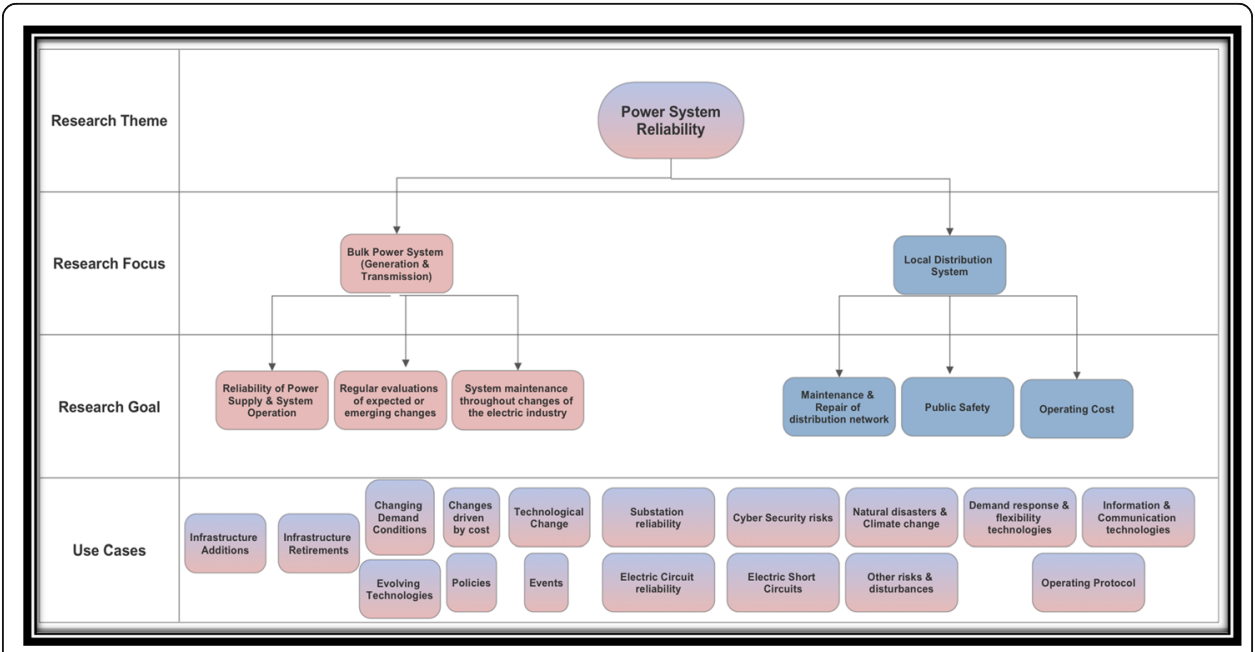

Fig. 2 Power-system reliability research framework 
The grid systems in Hawaii and Alaska are not connected to the grids in the continental states (U.S. Department of Energy, Office of Electricity 2015).

In the context of the power-system reliability research theme, possible scenarios, or use cases, can be classified into: infrastructure addition, infrastructure retirement, changing demand conditions, evolving technologies, changes driven by cost, policies, technological change, events, substation reliability, electric-circuit reliability, electric short circuits, cybersecurity risks, natural disasters and climate change, demand response and flexibility technologies, information and communication technologies, operating protocol, and other risks and disturbances.

\section{Foundational papers on the subject of electric-grid reliability}

One way to grasp the main core of a subject is to look at the references cited in the current articles and highlight papers that are continually referenced. This step was particularly helpful in identifying the foundational papers.

The same search string was used without an exclusion criterion (no year range restriction) to pull all journal and magazine publications from the database. The results (366 extracted articles) were sorted in descending order based on the number of citations (the number of other papers' reference lists that included them), the standard deviation for each article's number of citations was calculated, and the outliers (articles whose number of citations was more one standard deviations) were identified. Based on this analysis, 59 foundational papers were identified. After excluding the irrelevant papers and those not meeting the quality criteria, 39 relevant papers remained (Table 3).

This section presents a bibliography and the analysis of foundational papers on the subject of electric-grid reliability (Table 4).

Table 5 summarizes the research methods identified in the foundational papers. Though modeling and simulation are dominant research methods within the literature, articles using analytical approaches seem to be gaining more attention considering how often they are cited.

Bearing in mind the research themes and the methods illustrated in the foundational papers, analytics has been a popular topic in research and more research is needed in the area of reliability planning and improvement, particularly in the energy field. Through the use of GIS, machine learning, and data-mining techniques, analytics would help the research community plan and improve the smart grid.

Table 3 Foundational paper statistics

\begin{tabular}{ll}
\hline Database criteria & Total count \\
\hline Journal and magazine papers found by the search & 366 \\
Standard deviation for the articles' citation counts & 30 \\
Articles whose \# of citations exceeded one standard deviation (30 citations) & 53 \\
Irrelevant papers & 10 \\
Papers not included due to inadequate quality & 4 \\
Final number of relevant anchor papers & 39 \\
\hline
\end{tabular}




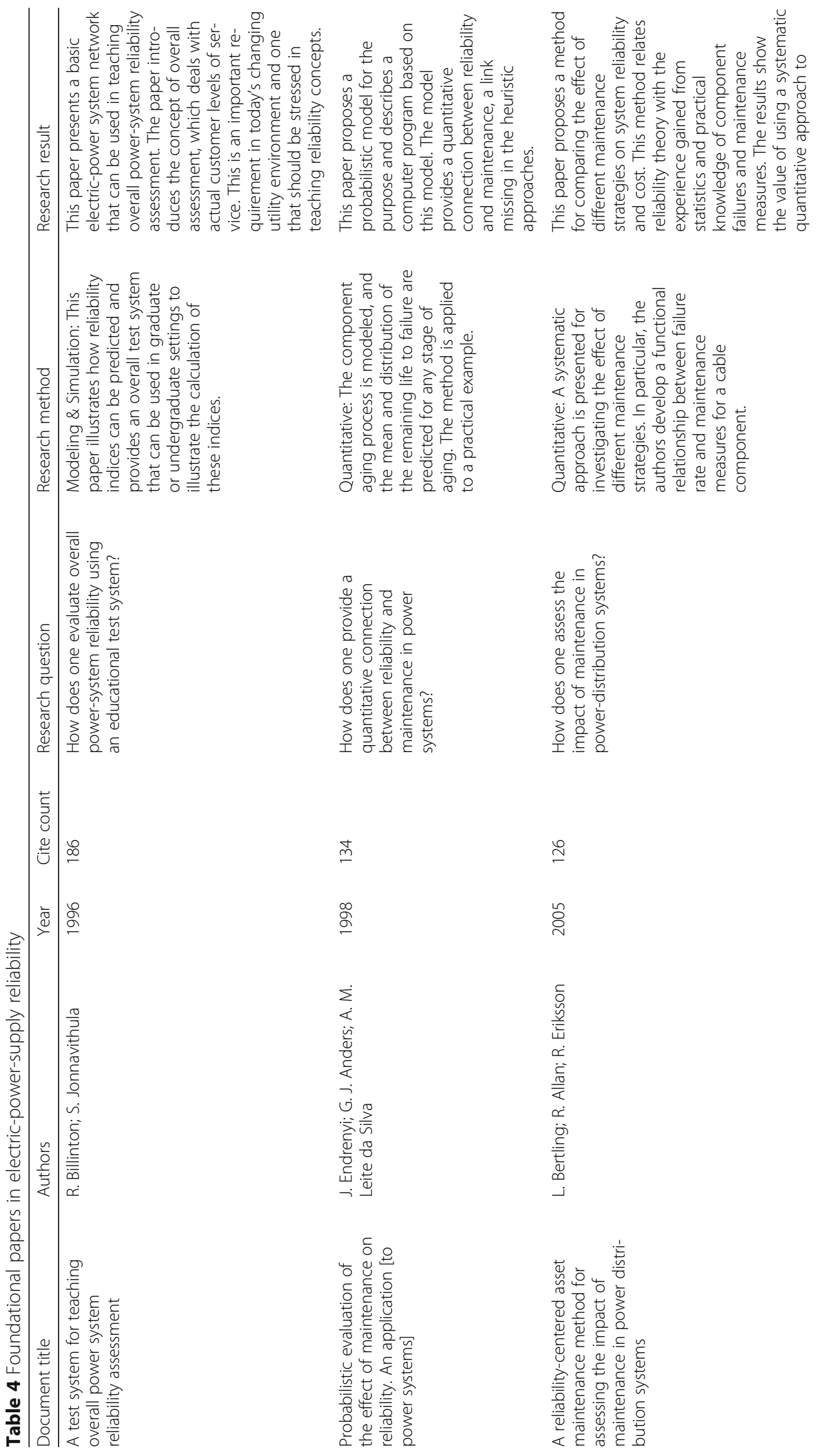




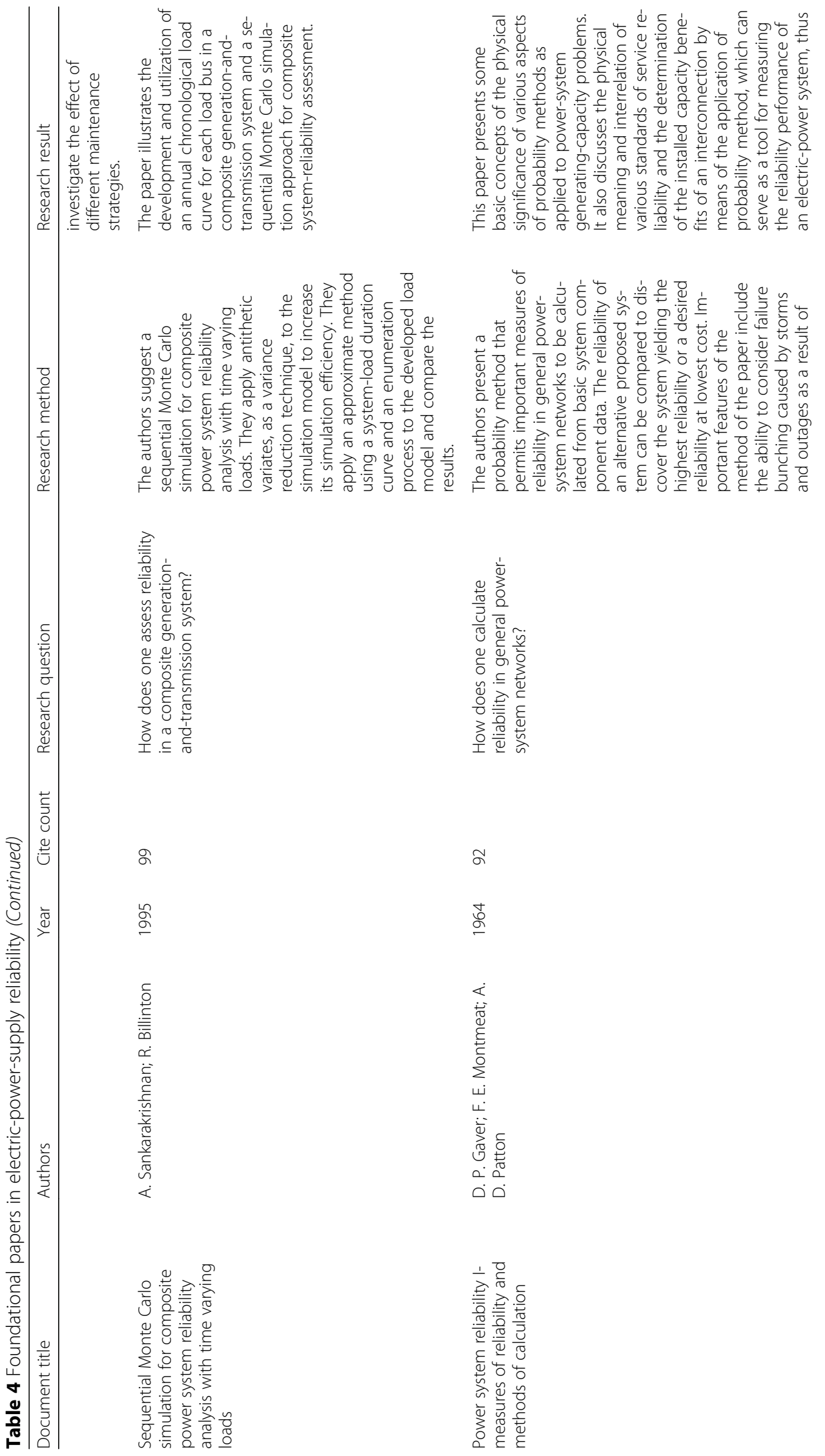




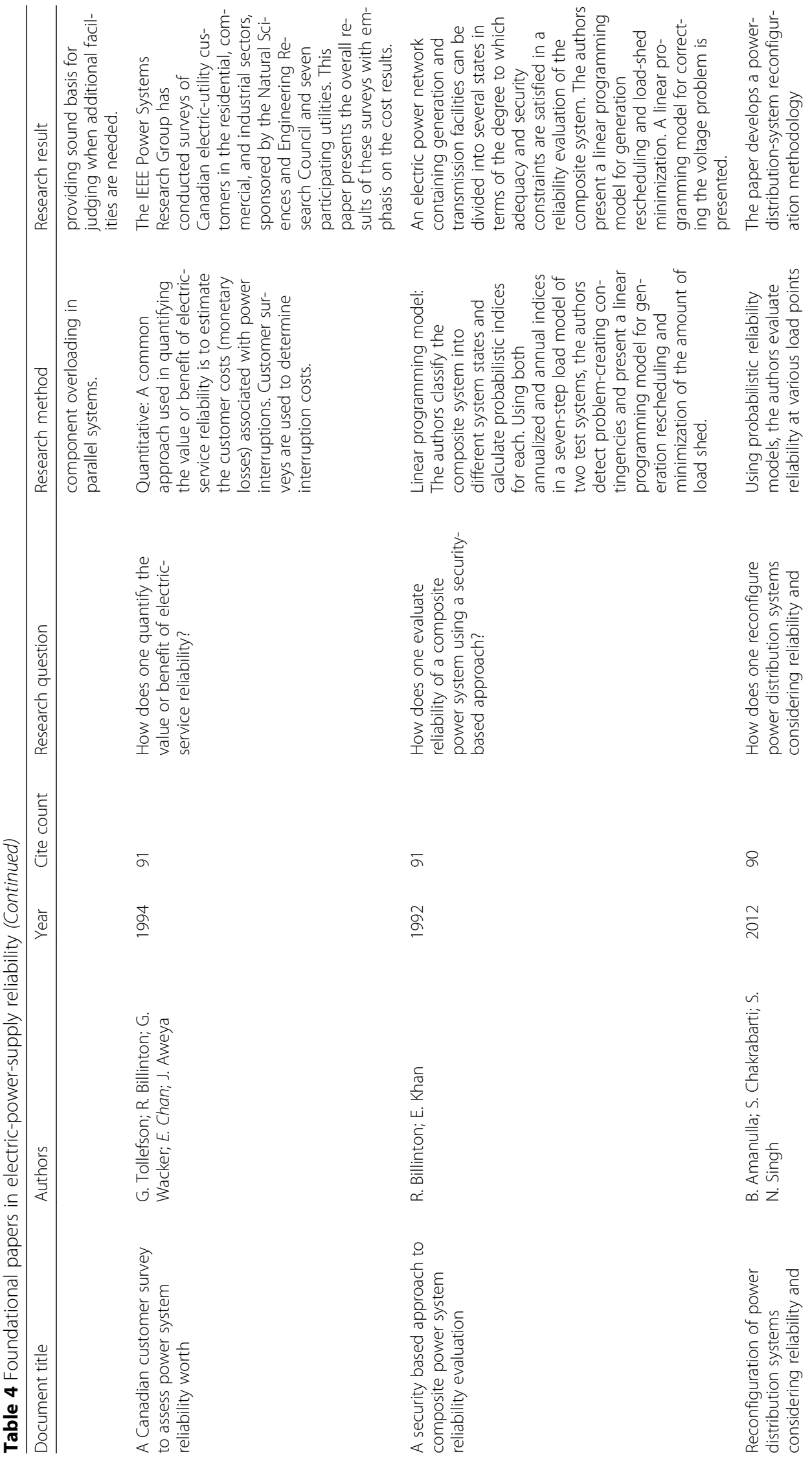




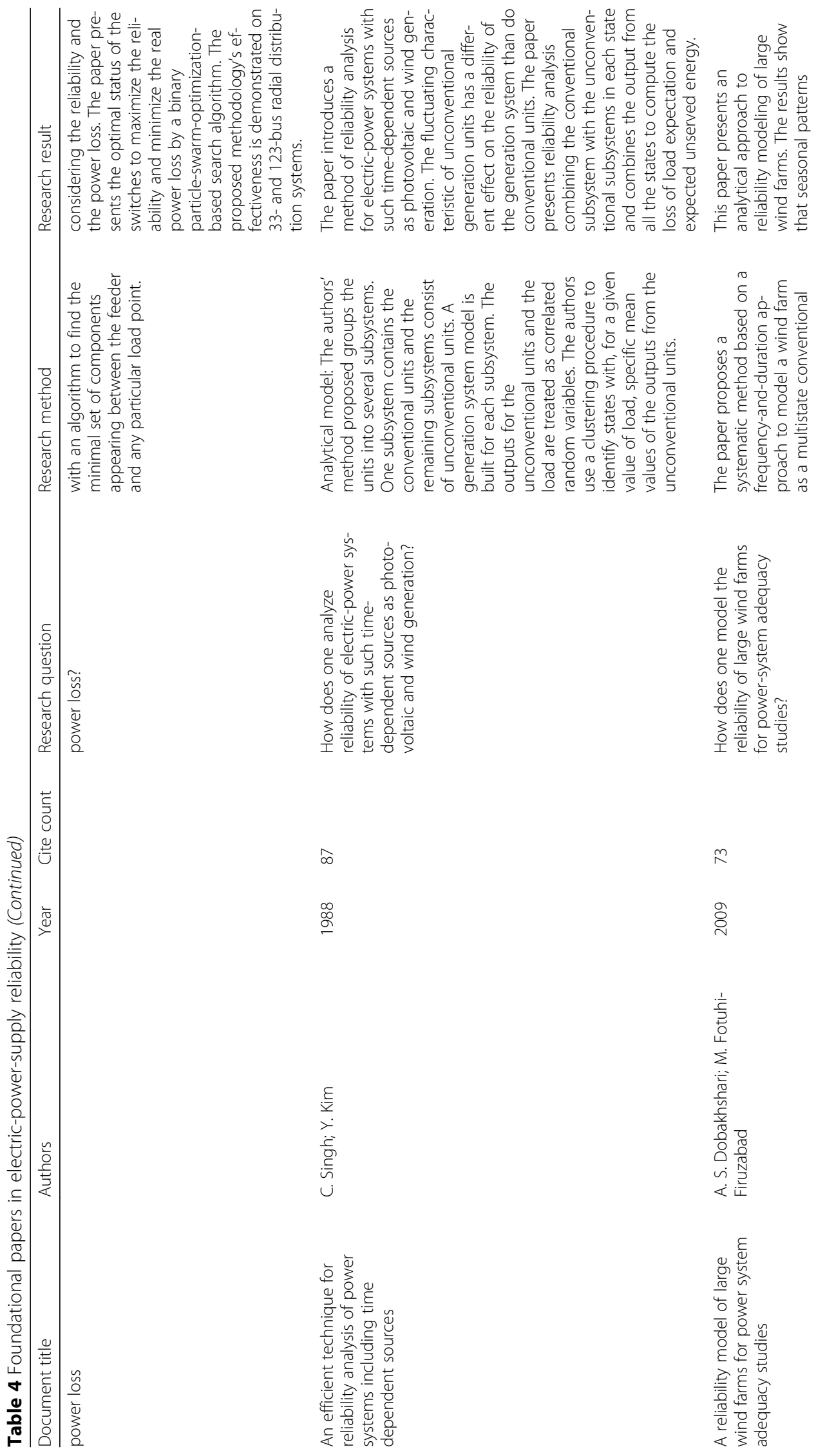




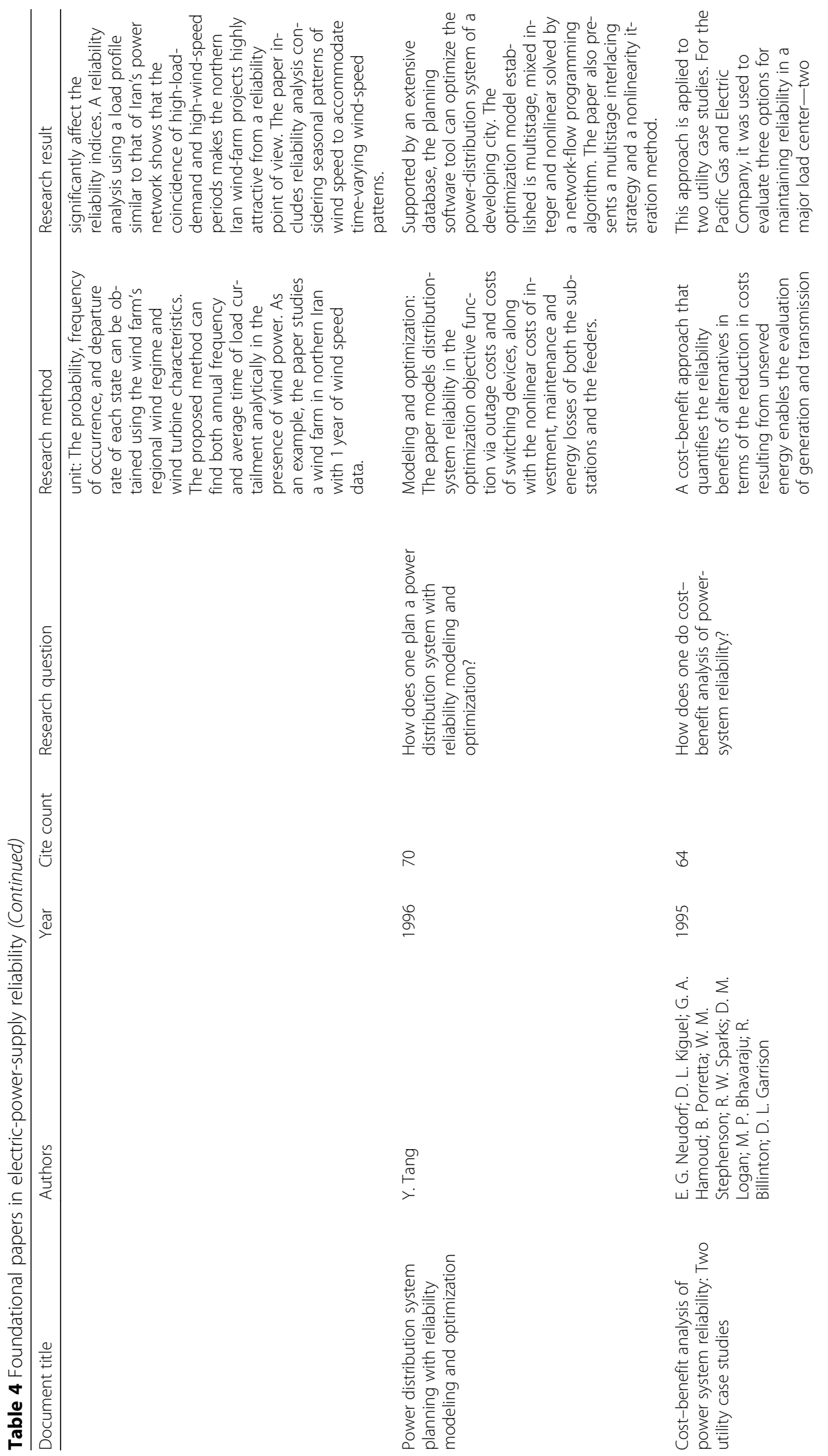




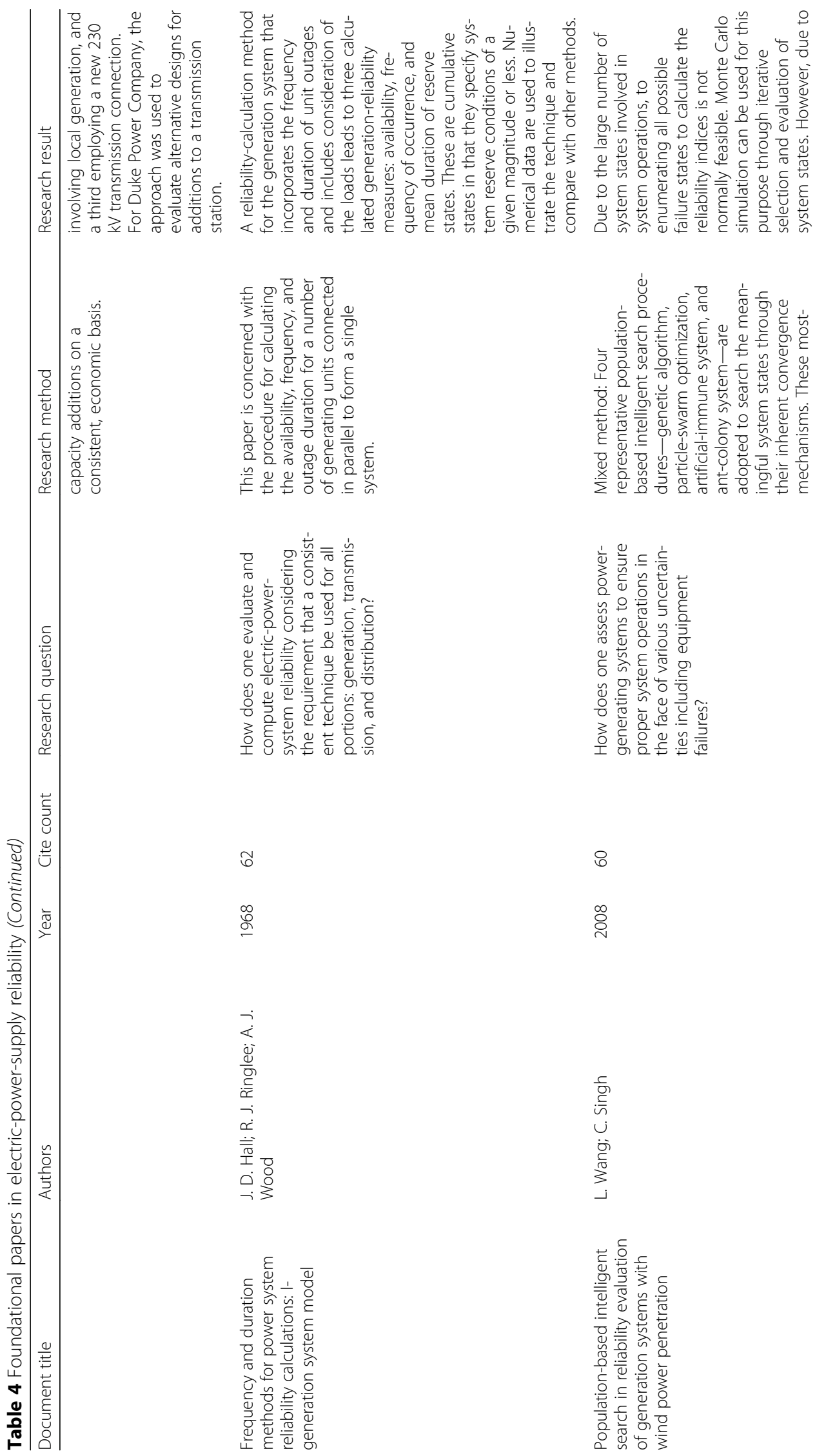




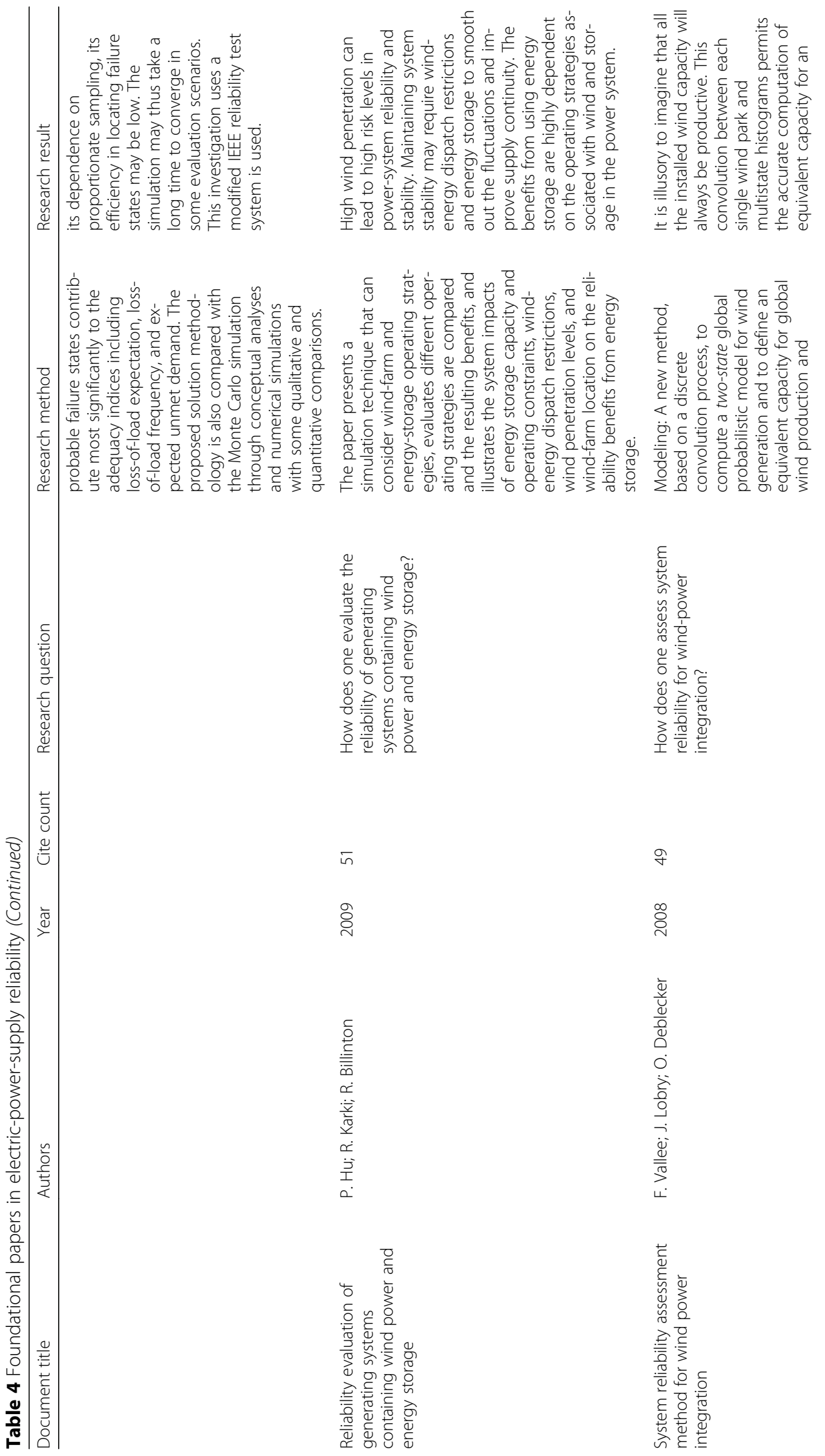




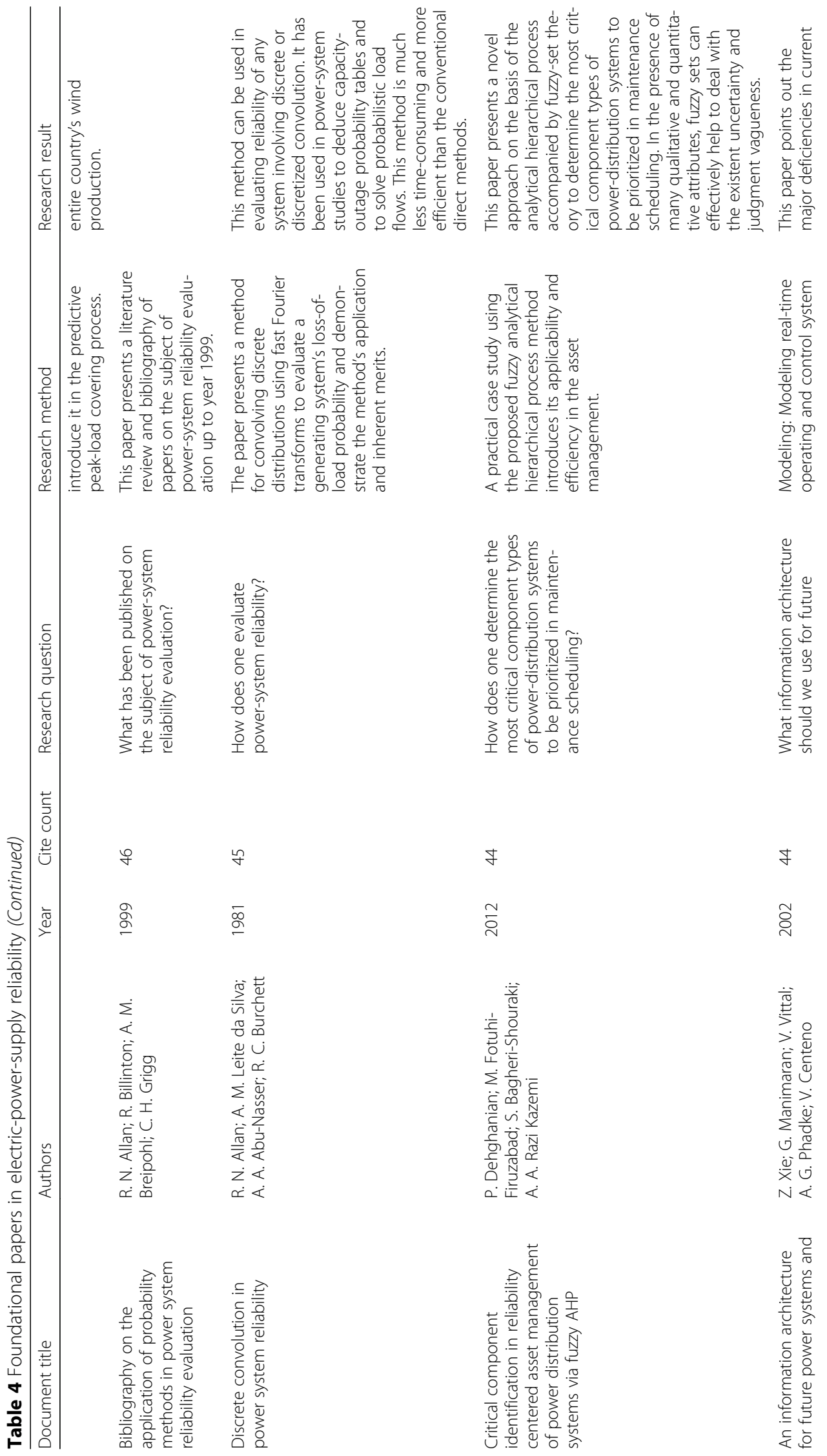




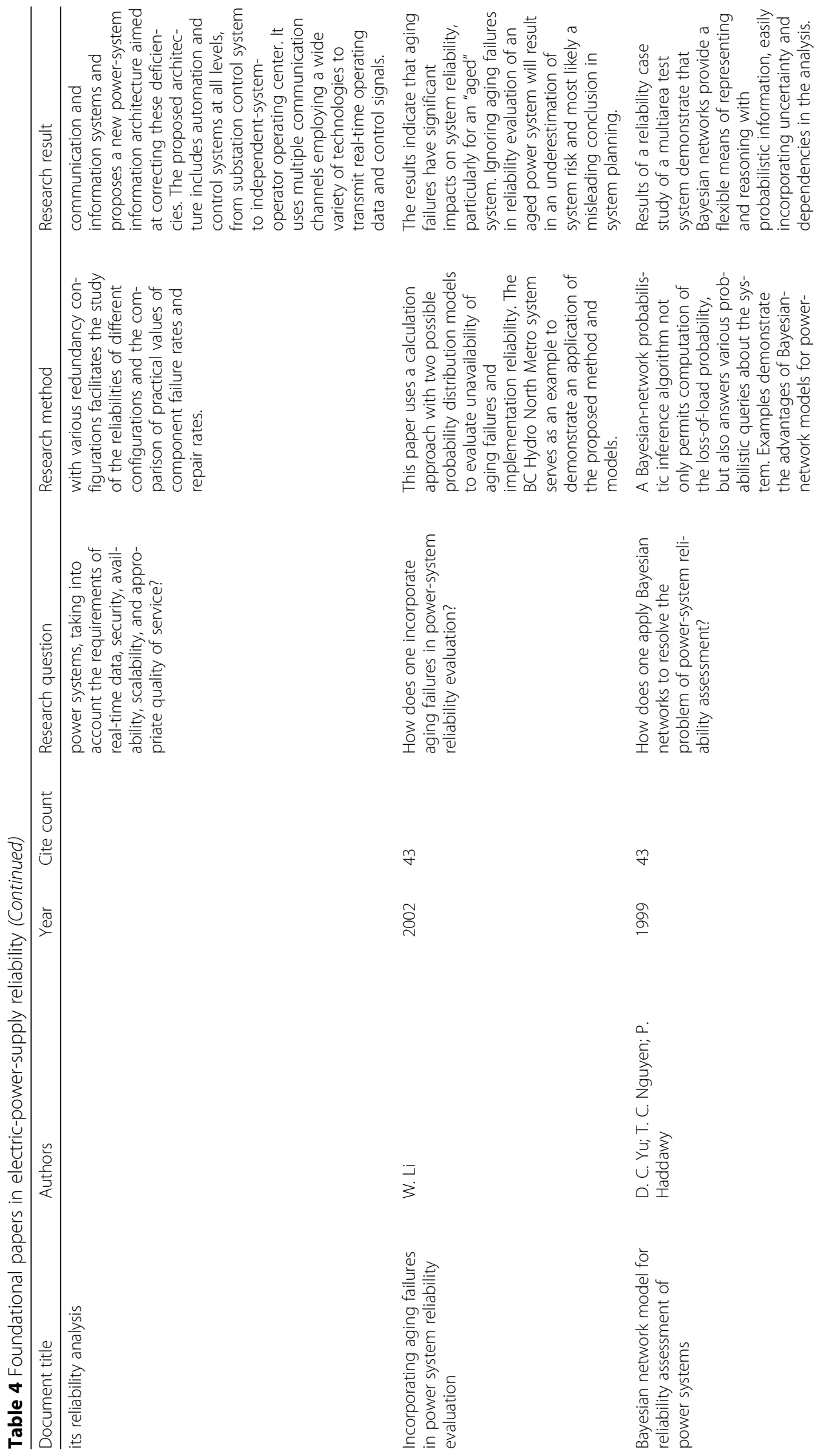




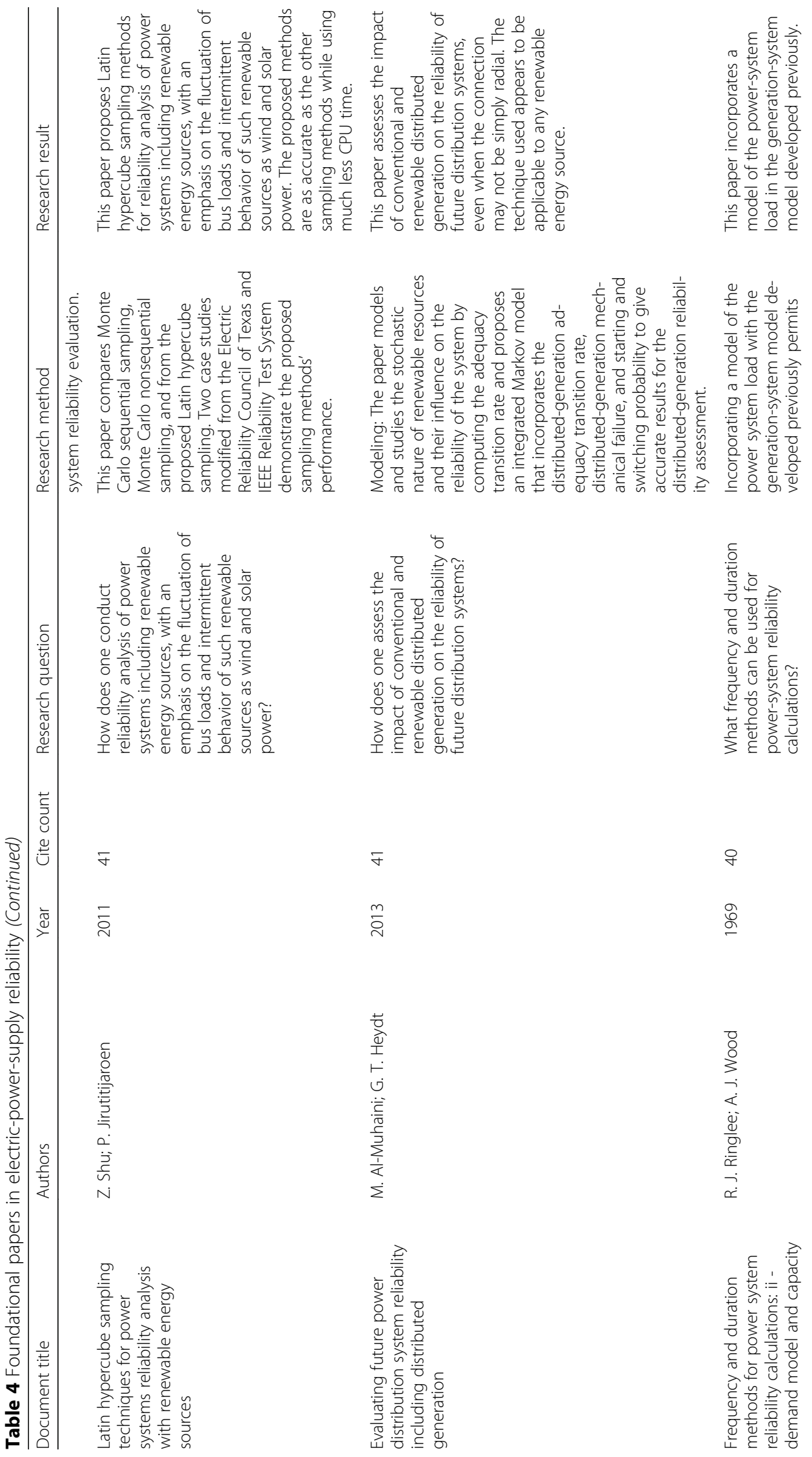




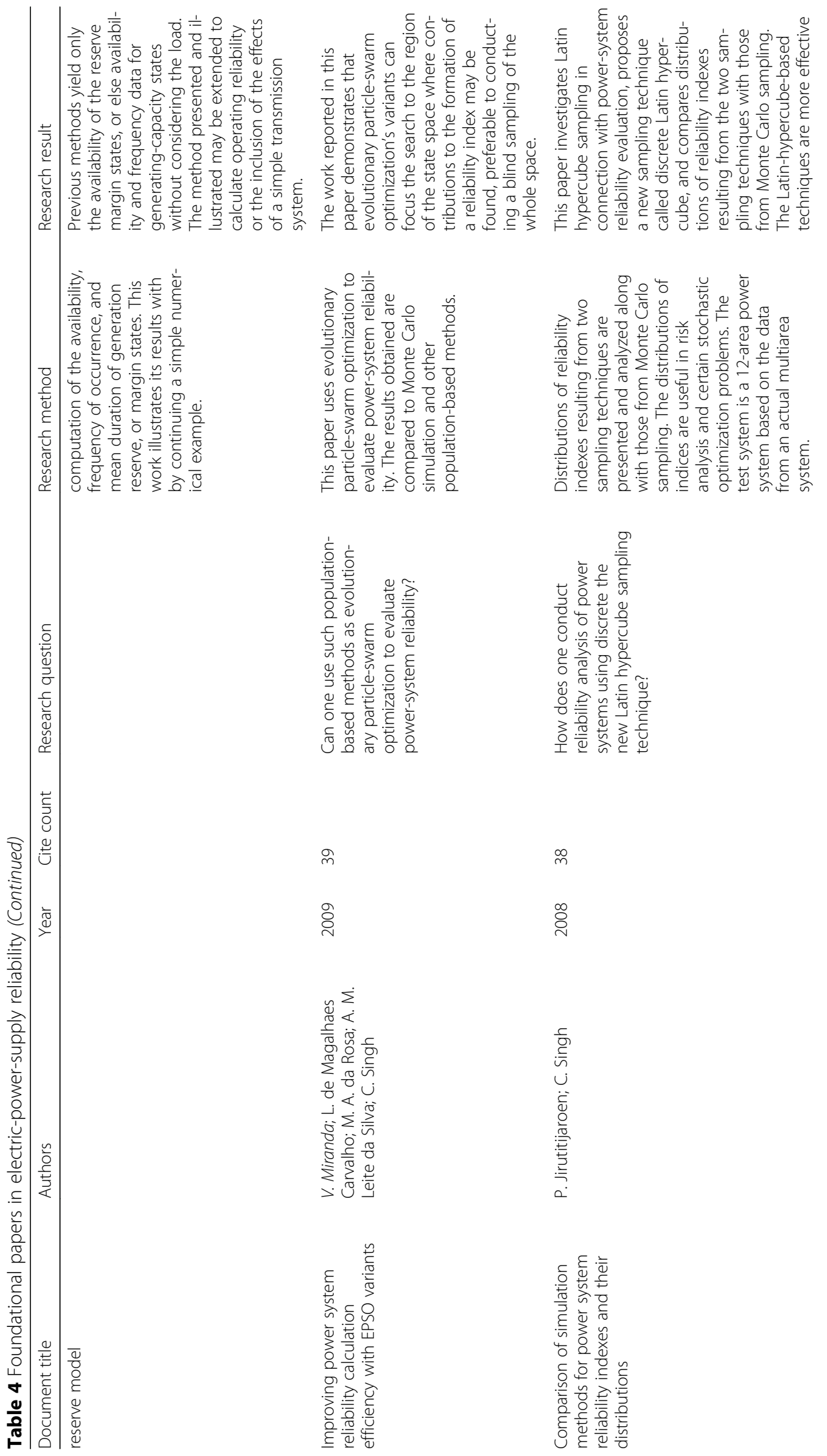




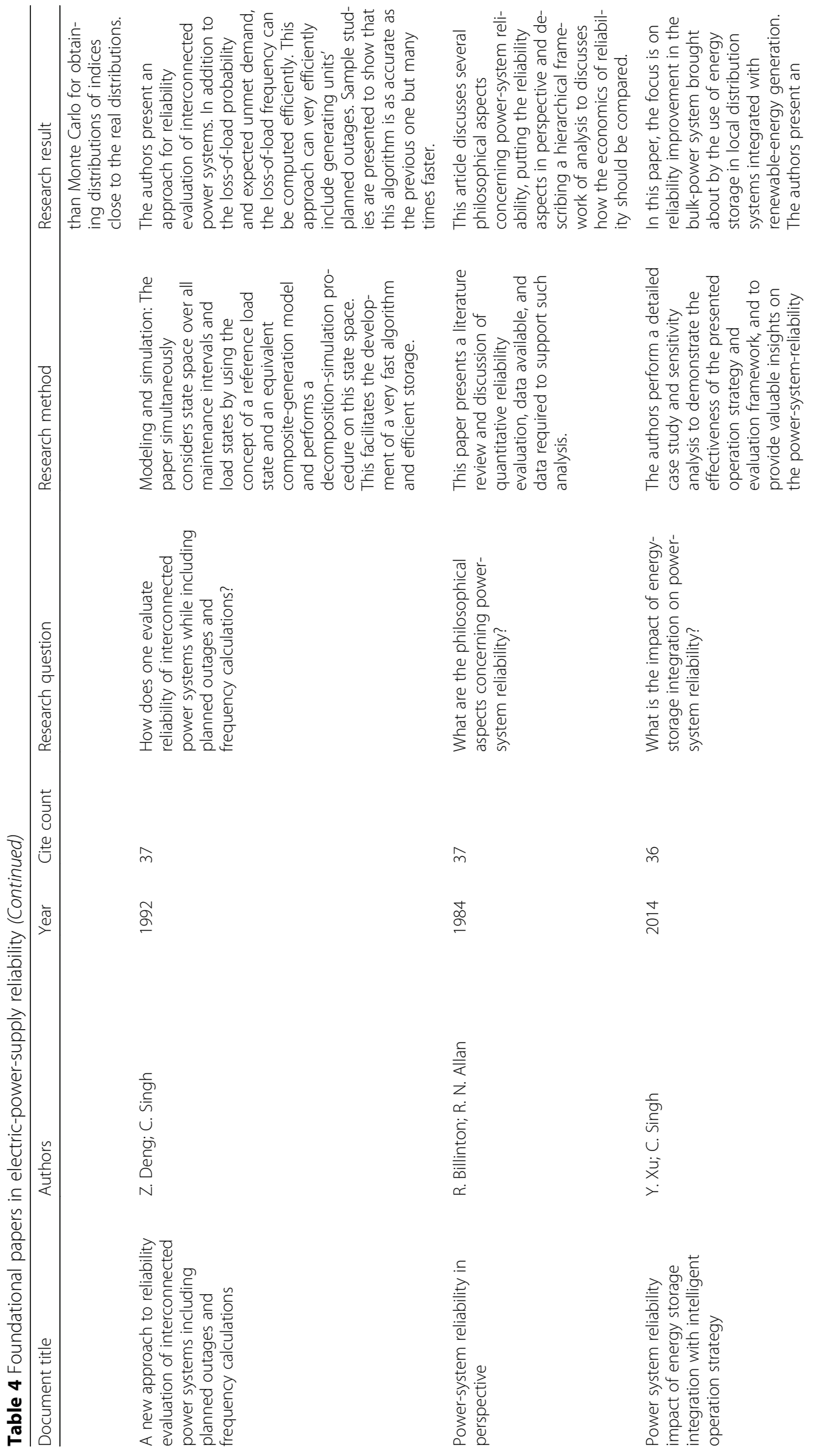




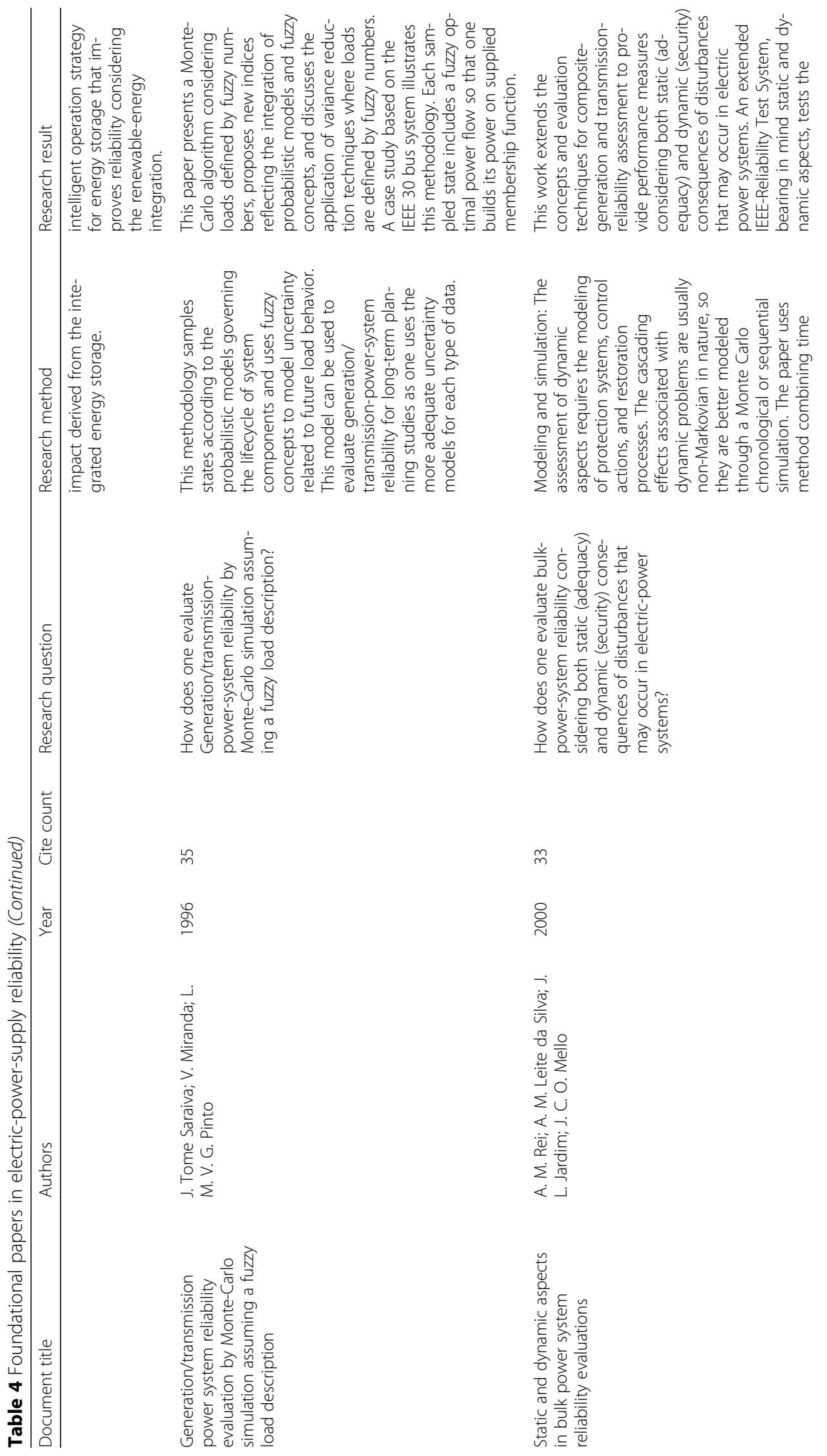




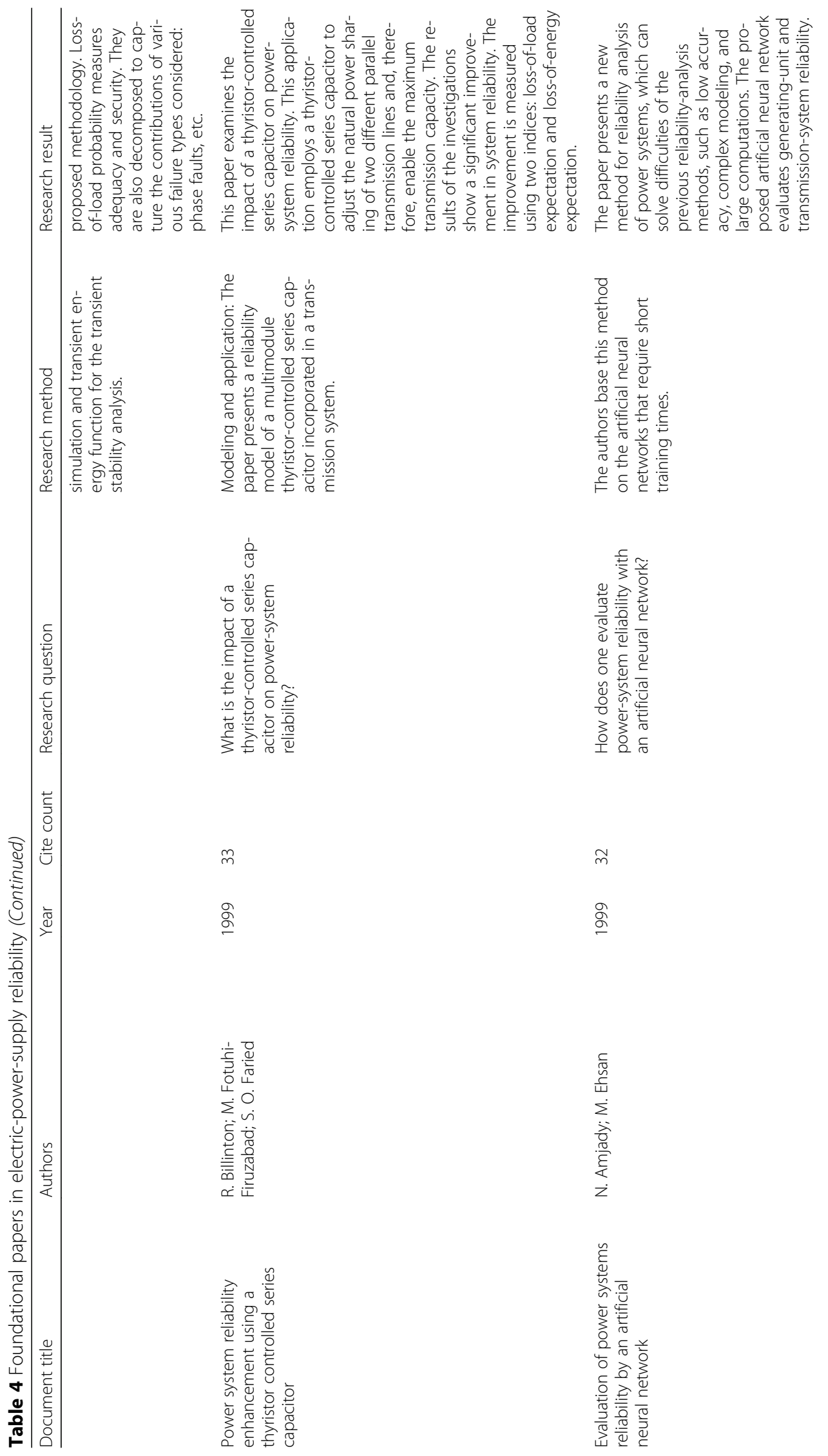




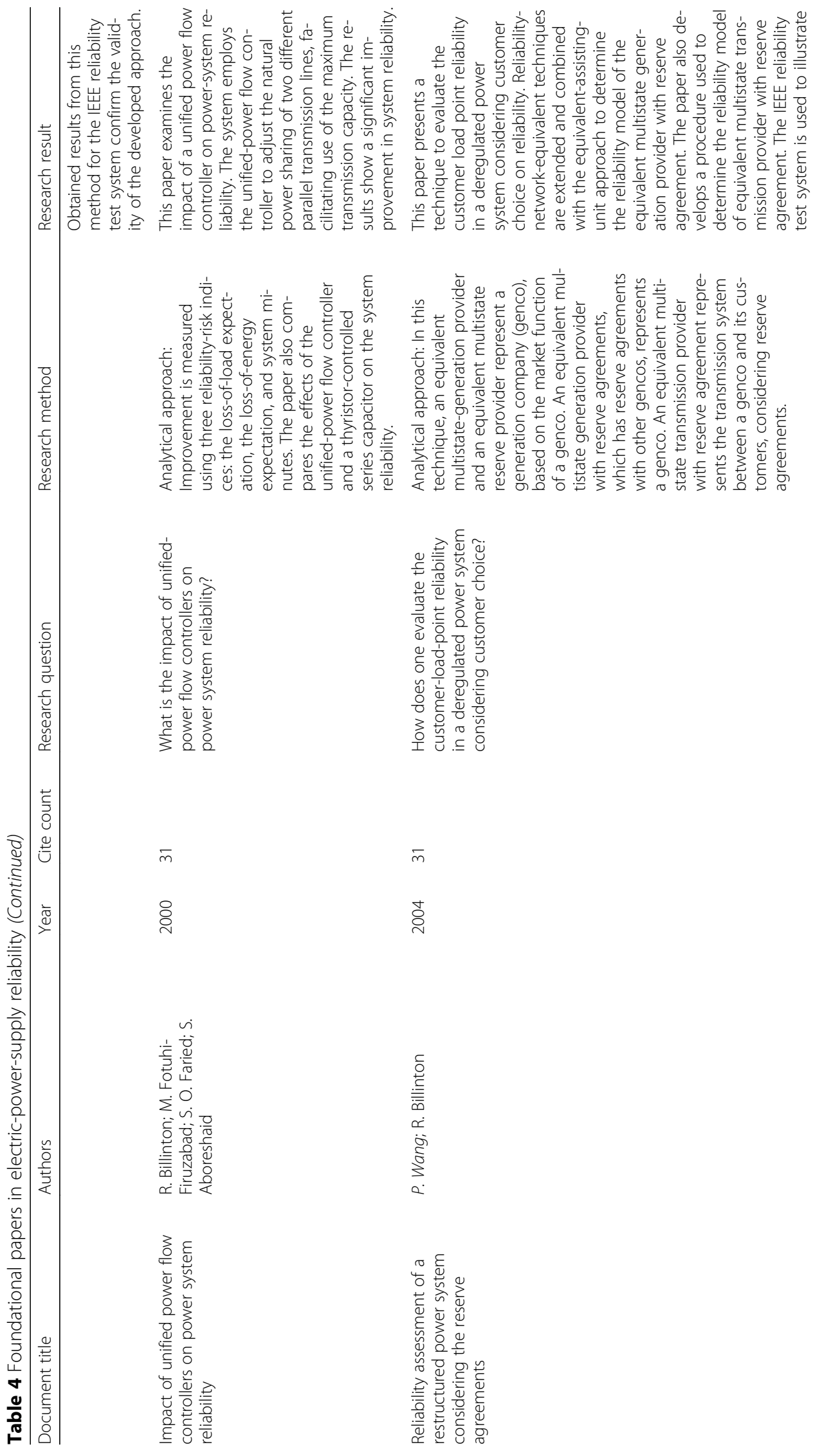




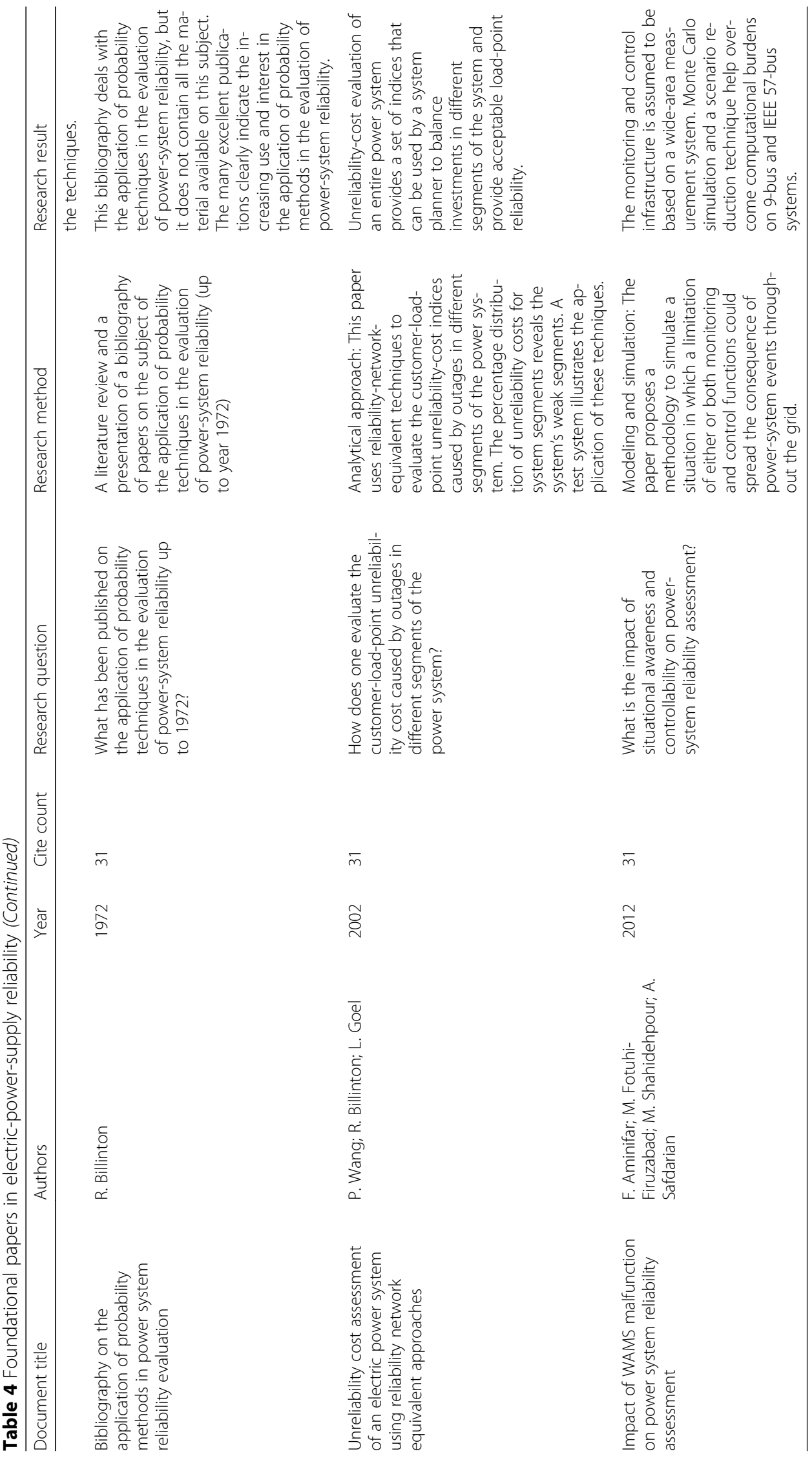


Table 5 Research methods

\begin{tabular}{lll}
\hline Research method & \multicolumn{2}{l}{ Count anchor paper used the method } \\
\cline { 2 - 3 } & $N$ & Percent \\
\hline Modeling \& simulation & 16 & 41 \\
Quantitative/Analytical approach & 20 & 51 \\
Literature review \& discussion & 3 & 8 \\
\hline
\end{tabular}

\section{Conclusion}

This paper identifies the use of analytics to predict reliability issues and plan for grid reliability. To engender cutting-edge grid-reliability research activity, prospective authors in this space could examine topics such as:

- Modeling grid behavior,

- Detecting outages,

- Location analytics on smart-grid resiliency,

- Planning secondary and tertiary backup for the smart grid,

- Data management and pipeline to enable analytics,

- Internet of things (IOT) and sensors for analytics, and

- Recent advances in analytics and smart grids.

This systematic literature review of grid-reliability research provides a solid foundation to equip researchers with the most pertinent information, offers an enhanced EI research framework, and provides directions for future research in this domain.

Abbreviations

BPS: Bulk-power system; El: Energy Informatics

Acknowledgements

The first author thanks her entire dissertation committee for their helpful advice.

Funding

This literature review is based on the first author's doctoral dissertation earned from Claremont Graduate University. No additional funding was used for this project.

Availability of data and materials

Additional supporting data is available from the first author.

Authors' contributions

The first author completed the literature review. The second author was the chair of the first author's dissertation committee, designed the paper structure, reviewed the findings, and proofread/edited the final paper. All authors read and approved the final manuscript.

Ethics approval and consent to participate

Not applicable.

Consent for publication

Not applicable.

Competing interests

The authors declare that they have no competing interests.

Publisher's Note

Springer Nature remains neutral with regard to jurisdictional claims in published maps and institutional affiliations. 
Received: 18 February 2019 Accepted: 12 April 2019

Published online: 02 May 2019

\section{References}

Adderly S (2016) Reviewing power outage trends, electric reliability indices and smart grid funding. Thesis, University of Vermont. http://scholarworks.uvm.edu/cgi/viewcontent.cgi?article=1530\&context=graddis. Accessed 15 Sept 2017

Campbell RJ (2012) Weather-related power outages and electric system resiliency. (Congressional Research Service Report, Vol. August 28, pp. 1-15). https://fas.org/sgp/crs/misc/R42696.pdf. Accessed 15 Sept 2017

Chayanam K (2005) Analysis of telecommunications power outages. Thesis. https://etd.ohiolink.edu/rws_etd/document/get/ ohiou1125024491/inline. Accessed 15 Sept 2017

Diesel Service and Supply (2017). The many causes of power failures. http://www.dieselserviceandsupply.com/Causes_of_ Power_Failures.aspx. Accessed 11 Sept 2017

Eto JH, Lacommare, KH, Larsen P, Todd A, Fisher E (2012) An examination of temporal trends in electricity reliability based on reports from U.S. electric utilities. https://emp.lbl.gov/publications/examination-temporal-trends. Accessed 15 Sept 2017

Goebel C, Jacobsen H-A, del Razo V, Doblander C, Rivera J, llg J et al (2014) Energy informatics-current and future research directions. BISE 6:25-31. https://doi.org/10.1007/s12599-013-0304-2

Hirst E, Kirby B (2000) Bulk-power basics: reliability and commerce. Consulting in Electric-Industry Restructuring, Oak Ridge http://www.consultkirby.com/files/RAPBPBasics.pdf

Kenward A, Raja U (2014) Blackout: extreme weather, climate change and power outages. https://www.eenews.net/assets/ 2014/04/14/document_ew_01.pdf. Accessed 15 Sept 2017

Kitchenham B (2004) Procedures for performing systematic reviews (NICTA Technical Report 0400011T.1). Durham University and Keele University, Durham http://www.inf.ufsc.br/ aldo.vw/kitchenham.pdf. Accessed 11 Sept 2017

Kitchenham B, Charters S (2007). Guidelines for performing systematic literature reviews in software engineering (Keele University and Durham University Joint Report). https://userpages.uni-koblenz.de/ laemmel/esecourse/slides/slr.pdf

Laezman R (2017) Office of Energy invests in grid reliability research. Electrical Contractor Magazine. http://www.ecmag.com/ section/systems/office-energy-invests-grid-reliability-research. Accessed 11 Sept 2017

Mitnick S (2015) Changing uses of the electric grid: reliability challenges and concerns. http://www.emrf.net/uploads/3/4/4/6/ 34469793/emrf_business_models_final_web_version.pdf. Accessed 11 Sept 2017

National Academies of Sciences, Engineering, and Medicine (2017) Enhancing the resilience of the nation's electricity system. The National Academies Press, Washington, DC. https://doi.org/10.17226/24836

Osborn J, Kawann C (2001) Reliability of the U.S. electricity system: recent trends and current issues. Energy Analysis Department, Ernest Orlando Lawrence Berkeley National Laboratory, Berkeley https://emp.lbl.gov/sites/all/files/ REPORTIbnl-47043.pdf. Accessed 11 Sept 2017

Profeta T (2017) The climate post: grid reliability study released as climate change panel disbands. http://www.huffingtonpost com/entry/the-climate-post-grid-reliability-study-released-as_us_599ef43ae4b0a62d0987ad61. Accessed 11 Sept 2017

Rocky Mountain Power (2017) Key causes of power outages. https://www.rockymountainpower.net/ed/po/or/kcopo.html. Accessed 11 Sept 2017

Spanos G, Angelis L (2016) The impact of information security events to the stock market: a systematic literature review. Comput Secur 58:216-229. https://doi.org/10.1016/j.cose.2015.12.006

U.S. Department of Energy (2013) Smart Grid Investment Grant Program progress report II. https://www.smartgrid.gov/files/ SGIG_progress_report_2013.pdf. Accessed 15 Sept 2017

U.S. Department of Energy (2015) Enabling modernization of the electric power system. In: Quadrennial technology review: An assessment of energy technologies and research opportunities http://energy.gov/downloads/chapter-3-enablingmodernization-electric-power-system. Accessed 11Sept 2017

U.S. Department of Energy, Office of Electricity (2015). United States electricity industry primer (DOE/OE-0017). https:/www. energy.gov/sites/prod/files/2015/12/f28/united-states-electricity-industry-primer.pdf. Accessed 11 Sept 2017

U.S. Department of Energy, Office of Energy Efficiency and Renewable Energy (2017) Performance and operation. https:// energy.gov/eere/sunshot/grid-performance-and-reliability. Accessed 11 Sept 2017

Watson RT, Boudreau M-C (2011) Energy informatics. Green ePress, Athens

Westar Energy (2017). What causes power outages? https://www.westarenergy.com/outage-causes. Accessed 11 Sept 2017

Wisconsin Public Service (2017) Why power outages occur. http://www.wisconsinpublicservice.com/home/power_occur.aspx. Accessed 11 Sept 2017

\section{Submit your manuscript to a SpringerOpen ${ }^{\circ}$ journal and benefit from:}

- Convenient online submission

- Rigorous peer review

- Open access: articles freely available online

- High visibility within the field

- Retaining the copyright to your article

Submit your next manuscript at $\boldsymbol{\nabla}$ springeropen.com 